\title{
PET Probe-Guided Surgery in Patients with Breast Cancer: Proposal for a Methodological Approach
}

\author{
PAOLO ORSARIA ${ }^{1}$, AGOSTINO CHIARAVALLOTI ${ }^{2}$, ALESSANDRO FIORENTINI $^{2}$, CHIARA PISTOLESE $^{2}$, \\ GIANLUCA VANNI ${ }^{1}$, ALESSANDRA VITTORIA GRANAI ${ }^{1}$, DIMITRIOS VARVARAS ${ }^{1}$, ROBERTA DANIELI $^{2}$, \\ ORAZIO SCHILLACI ${ }^{2}$, GIUSEPPE PETRELLA ${ }^{1}$ and ORESTE CLAUDIO BUONOMO ${ }^{1}$ \\ ${ }^{1}$ Department of Surgery, Tor Vergata University Hospital, Rome, Italy \\ ${ }^{2}$ Department of Biomedicine and Prevention, Tor Vergata University Hospital, Rome, Italy
}

\begin{abstract}
Background: Although it is valuable for detecting distant metastases, identifying recurrence, and evaluating responses to chemotherapy, the role of ${ }^{18} \mathrm{~F}$-fluorodeoxyglucose positron-emission tomography/computed tomography $\left({ }^{18} \mathrm{~F}-\mathrm{FDG}\right.$ $P E T / C T)$ in assessing locoregional nodal status for initial staging of breast cancer has not yet been well-defined in clinical practice. In the current report, we describe a new PET probebased clinical approach, with evaluation of the technical performance of a handheld high-energy gamma probe for intraoperative localization of breast carcinomas, and evaluation of lymph node metastases during radio-guided oncological surgery. Patients and Methods: Three patients underwent a PET/CT scan immediately prior to surgery following the standard clinical protocol. Intraoperatively, tumors were localized and resected with the assistance of a hand-held gamma probe. PET-guided assessment of the presence or absence of regional nodal spread of malignancy was compared with the reference standard of histopathological examination. Results: In all three cases, perioperative ${ }^{18}$ F-FDG PET/CT imaging and intraoperative gamma probe detection verified complete resection of the hypermetabolic lesions and demonstrated no additional suspicious occult disease. Conclusion: This innovative approach demonstrates great promise for providing real-time access to metabolic and morphological tumor information that may lead to an optimal disease-tailored approach. In carefully selected indications, a PET probe can be a useful adjunct in surgical practice, but further trials with a larger number of patients need to be performed to verify these findings.
\end{abstract}

This article is freely accessible online.

Correspondence to: Paolo Orsaria, Department of Surgery, Tor Vergata University Hospital, 81 Oxford Street, 00133 Rome, Italy. Mobile: +39 3282492485, e-mail: orsaria@aol.it

Key Words: Breast cancer, nodal staging, sentinel node, positronemission tomography (PET) probe, radio-guided surgery.
Nuclear medicine has an essential and increasing role in the management of patients with breast cancer. Sentinel node detection has achieved reliability and, at present, it has an important place in clinical management. Even in the era of gene-expression profiling, nodal status remains one of the primary prognostic discriminants in patients with breast cancer, and is of great value as an independent predictor of distant disease development. Although several institutional case series have differed in patient selection, follow-up, type of surgery, and adjuvant therapies, they have consistently shown that the percentage of positive lymph nodes is a significant indicator of survival in women with axillary metastases on final pathology (1-5). The American Society of Clinical Oncology convened an Update Committee of experts to provide evidence-based recommendations to practicing oncologists, surgeons, and radiation therapy clinicians on the use of sentinel node biopsy (SNB) for patients with early-stage breast cancer (6). The need to perform axillary lymph node dissection (ALND) when SNB is positive and administration of contemporary adjuvant treatment including radiotherapy, chemotherapy, and hormonal therapy has been questioned in recent years. On the other hand, ongoing trials are testing whether node-positive patients can be spared chemotherapy, as qualifying indications increasingly rely on breast cancer biology for decision-making regarding adjuvant systemic treatment (7). In summary, sentinel node surgery represents the next major step in reducing the extent of surgical procedures, but despite the revolution of quality-conserving care, recent data collection and analyses of anatomical techniques suggest that exact lymphatic drainage of the breast is debatable (8). Different lymphatic patterns may help explain some important unresolved clinical problems, including different detection rates between studies and high false-negative rates of about $10 \%$ in multicenter randomized controlled trials (9). Further anatomical investigation and knowledge of the exact sentinel lymphatic channels will provide more information about patterns of breast cancer in order to improve surgical strategy, locoregional recurrence, and survival. 
Many authors have acknowledged the value of positronemission tomography (PET) with ${ }^{18} \mathrm{~F}$-2-deoxy-2-fluoro-Dglucose $\left({ }^{18} \mathrm{~F}\right.$-FDG) in the differential diagnosis of breast lesions, and in locoregional staging, since breast cancer is strongly avid for glucose (10). A positive correlation was found between ${ }^{18}$ F-FDG uptake and tumor histology, microscopic tumor growth patterns, and tumor cell proliferation. It should be noted that metabolic changes after malignant transformation depend on many complex interactions between cellular metabolism and the tumor microenvironment (11). For these reasons, the published results are heterogeneous, but show very clearly that ${ }^{18} \mathrm{~F}$-FDG-PET is a non-invasive in vivo method of providing information that can be of prognostic interest, and is related to overall and disease-free survival (12). Techniques that are primarily physiological may, therefore, have a key role to play in the adjunctive assessment of the breast, especially when there is doubt over the validity of anatomical imaging. In addition, despite advances in diagnostic assessment using PET and the availability of new systemic treatment options, the treatment paradigm in oncology has shifted towards more aggressive therapeutic interventions, including cytoreductive techniques and metastasectomy. Intraoperative localization of PET-positive recurrent or metastatic lesions can be facilitated using a hand-held PET probe, thus improving the success of surgical exploration in selected indications (13). Locating a lesion that is not easily seen or palpated during routine surgical exploration is probably the strongest indication for PET probeguided procedure. The patients most suitable for this technique are those presenting with non-palpable, but FDG-PET-positive lymph nodes in axilla, where surgical dissection is relatively more challenging than that of the groin or neck. Therefore, we propose an innovative approach for intraoperative tumor localization and axillary staging utilizing ${ }^{18} \mathrm{~F}-\mathrm{FDG}$ for perioperative PET/CT imaging and intraoperative gamma probe detection to verify complete resection of hypermetabolic lesions in patients with breast cancer. Such guided axillary sampling could represent a potential further development in recent anatomical and functional methods, offering a dynamic technique to be used according to clinical and anatomical disease parameters (14). Furthermore, it is important to identify appropriate inclusion criteria for PET probe-guided surgery, taking into consideration whether distinct molecular traits might be predictive of different nodal involvement and outcomes (15). Given the apparent heterogeneity of subgroups within breast cancer subtypes, and different genomic features, this ability will also contribute to the functional evaluation of lymph nodes in the detection of sub-clinical metastases. Our review of data revealed that the probability of having an axillary metastasis seemed to be more frequently related to the luminal breast cancer group [luminal A, luminal B (human epidermal growth factor receptor 2 negative (HER2 ${ }^{-}$) luminal B HER $2^{+}$) with a three times higher risk for patients with luminal B HER2 ${ }^{-}$breast cancer compared with HER2 ${ }^{+}$(15).
This is in contrast to radiological criteria, that are based solely on anatomical findings. Prospective database analysis and improved molecular profiling techniques will also likely provide direction in the management of this increasingly frequent clinical problem.

In the current preliminary case reports, whose data were extracted from a prospective study that is currently in progress at our Department (see below), we describe a new PET probebased clinical approach. We evaluated the technical performance of a handheld high-energy gamma probe in the diagnosis of breast cancer and lymph node metastasis during radio-guided oncological surgery. Relationships between the intrinsic biological properties of the primary tumor, prognostic factors, and the axillary status of patients with breast cancer will be taken into account for a preliminary comparison between real-time intra-procedural PET/CT nodal detection and traditional staging procedures in terms of survival outcomes. The clinical use of PET probes has been limited to clinical trial settings, and no standard PET probe-guided surgery protocol has been developed.

\section{Patients and Methods}

Study design and conduct. This pilot study with interventional and non-pharmacological design used the following inclusion criteria. Patients were eligible if they were older than 18 years, had a histological diagnosis of invasive breast cancer within 3 months, had a primary tumor deemed technically appropriate for surgical resection, and had no clinical evidence of regional nodal or distant metastatic disease. Patients with co-morbidities (i.e. cardiovascular disease) were also considered.

The study protocol was approved by the Tor Vergata University Hospital Institutional Ethic Board (approval number 52.15, and each patient gave their informed consent to participation.

Three patients underwent a preoperative whole-body ${ }^{18}$ FDG-PET scan. PET assessment of the presence or absence of regional nodal spread of malignancy was compared with the reference standard of axillary nodal assessment, a histological examination of resected axillary lymph nodes obtained by SNB alone, by SNB and ALND, or by ALND alone.

Patients were required to be willing to proceed with SNB or ALND after PET completion, and to undergo a level I/II ALND after SNB if the SNB was positive, or if the PET scan showed increased uptake in the ipsilateral axilla.

PET/CT scan protocol. All patients fasted for at least 5 hours before intravenous ${ }^{18} \mathrm{~F}-\mathrm{FDG}$ injection and all serum glucose levels were normal $(\leq 107 \mathrm{mg} / \mathrm{ml})$. On the morning of the planned surgery, patients were intravenously injected with $370-450 \mathrm{MBq}$ of ${ }^{18} \mathrm{~F}-\mathrm{FDG}$ and hydrated with $500 \mathrm{ml}$ of intravenous saline $(0.9 \%$ sodium chloride). Physical activity was kept to a minimum with a rest period of 60 minutes post-injection. Images were obtained 60 minutes after FDG administration.

The PET/CT system Discovery ST16 (GE Medical Systems, Fairfield, CT, USA) was used for the whole cohort under examination. The system combines a high-speed ultra 16-detectorrow (912 detectors per row) CT unit and a PET scanner with 10,080 bismuth germanate crystals in 24 rings. The axial full width half 
maximum $1 \mathrm{~cm}$ radius is $5.2 \mathrm{~mm}$ in 3-dimensional (3D) mode and the axial field of view is $157 \mathrm{~mm}$. For the PET/low-dose computed tomography (ldCT) a low-amperage CT scan was acquired for attenuation correction of PET images $(80 \mathrm{~mA}, 140 \mathrm{kV}$, field of view about $420-500 \mathrm{~mm}$, and CT slice thickness of $3.75 \mathrm{~mm}$ ). The CT dose index for ldCT was $4.0175( \pm 0.84) \mathrm{mGy}$ and the dose-length product was $473.296( \pm 161.09) \mathrm{mGy}-\mathrm{cm}$. After non-enhanced CT, total-body PET examination in the caudocranial direction from the upper thighs to the vertex was performed (3.5 minutes per bed). Reconstruction was performed using the $3 \mathrm{D}$ reconstruction method of ordered subset expectation maximization with 30 subsets and two iterations

Images were reviewed by a nuclear physician and a surgical oncologist. Standard uptake values (SUV) and metabolic tumor volume (MTV) of the target lesion(s) were measured.

Evaluation of PET/CT images. PET/CT images were reviewed by two nuclear medicine physicians (AF and AC) on a dedicated PET/CT workstation (Advantage 4.4 and Xeleris 2; GE Healthcare, Fairfield, CT, USA), which allowed visualization of the PET and CT images separately or in fusion mode in the axial, coronal and sagittal planes. Uptake was considered pathological when an area of focal tracer uptake higher than the background was visually detected. Maximum SUV (SUVmax) were also determined and taken into account but no absolute cut-off value was used for the diagnosis. In cases in which there was disagreement between the readers, the patients were re-examined and a consensus was reached (16).

PET probe-guided surgery protocol. All patients underwent an ${ }^{18} \mathrm{~F}-$ FDG-PET study following the clinical protocol. The PET probeguided surgery decision was made after careful review of the imaging information and the clinical indications at a multidisciplinary meeting. Patients with unresectable tumors or evidence of distant metastases on the basis of PET/CT findings were excluded from the study.

Surgical exploration was scheduled 2-3 hours post-injection of the radiopharmaceutical. No glucose-containing intravenous fluids were allowed prior to or during the operation. Relevant images/views were available for viewing in the operating theatre.

A high-energy gamma probe with a gadolinium oxyorthosilicate crystal and $12.5 \mathrm{~mm}$ tungsten shielding (Care Wise Medical, Morgan Hills, CA, USA) were used. The analyzer was set for a photopeak of $511 \mathrm{keV}, \mathrm{a}$ window of $20 \%$, and a threshold of $490 \mathrm{keV}$. Calibration of the system and appropriate settings were verified prior to each operation. The probe and its connecting cord were placed in a plastic sleeve. Surgical exploration commenced with determination of the probe survey field. The initial probe survey was performed using the count per second mode. A target to background ratio (TBR) of 1.5 and above was used for confirmation of the target localization.

Technical considerations. ${ }^{18} \mathrm{~F}-\mathrm{FDG}$ PET is accepted as a useful tool in the preoperative evaluation and staging of appropriately selected patients with breast cancer, and there are currently some data available on the utilization of a handheld gamma probe for intraoperatively detecting various ${ }^{18} \mathrm{~F}-\mathrm{FDG}$-positive malignancies. However, there has been very little clinical investigation into specifically utilizing a combined approach of perioperative ${ }^{18}$ F-FDG PET technology and intraoperative ${ }^{18} \mathrm{~F}-\mathrm{FDG}$ gamma probe detection $(17,18)$. Intraoperative PET probe performance, as a general rule, is a function of radiopharmaceutical uptake, clearance kinetics, and probe engineering, all ultimately determining the TBR and detection threshold.
Regarding future planned studies and their data availability, it is important to realize that the success of PET probe-guided surgery depends on numerous factors, including the FDG avidity of the tumor, timing of surgical exploration in reference to injection of FDG, anatomical location of the lesion, its relative proximity to the main sites of physiological uptake, and technical properties of the probe. Data suggest that TBR improves over time up to 4 hours post-FDG administration (13). FDG metabolism and clearance occurs at a much faster rate in normal tissues than tumor tissue, and thus TBR improves with time, resulting in better lesion detection when imaging is delayed (19). Tumor-to-non-tumor and tumor-toorgan ratios are higher for delayed images than for the 1.5-hour routine images, and lesion detection is improved in nodal and hepatic metastases (20). Satisfactory count rates and TBR for surgical detection can be obtained 2-4 hours post-FDG administration, which makes this technique clinically feasible (13).

FDG avidity is determined by the glycolytic activity of the tumor and the viable tumor concentration in a given lesion. This feature contributes to the success of detection; however, it has nothing to do with specificity. Individual cancer types may also exhibit significant variability in terms of FDG avidity. Even in the same patient, different lesions may have different degrees of FDG uptake. The probe detects any FDG-avid lesion, whether it is malignant or inflammatory. Background radiation tends to decrease while tumor uptake is retained. The in situ TBR is also strongly affected by areas of physiological uptake or accumulation. The brain, cardiac and kidney uptake, and accumulation inside the bladder in the abdomen and pelvis affect the in situ TBR. Areas of physiological uptake show attenuation over time, and the use of intra-operative bladder catheterization minimizes interference from bladder accumulation of FDG.

Classifications of groups and staging. We identified intrinsic breast cancer subtypes, according to the clinicopathological criteria recommended by the St. Gallen International Expert Consensus Report 2013 (21). Patients were categorized based on the receptor status of their primary tumor as follows: luminal A [estrogenreceptor-positive $\left(\mathrm{ER}^{+}\right)$or progesterone-receptor-positive $\left(\mathrm{PR}^{+}\right)$, and HER2 ${ }^{-}$]; luminal B HER2 $2^{-}\left(\mathrm{ER}^{+}, \mathrm{HER} 2^{-}\right.$and at least one of $\mathrm{Ki}-67$ high or $\mathrm{PR}^{-}$or low); luminal $\mathrm{B}$ HER2 ${ }^{+}\left(\mathrm{ER}^{+}, \mathrm{HER} 2\right.$ overexpressed or amplified, any Ki-67, any PR); HER2 ${ }^{+}\left(\mathrm{ER}^{-}\right.$or $\mathrm{PR}^{-}$, and $\left.\mathrm{HER} 2^{+}\right)$, and basal $\left(\mathrm{ER}^{-}\right.$or $\mathrm{PR}^{-}$, and $\left.\mathrm{HER} 2^{-}\right)$. ER and PR status was determined using immunohistochemistry (IHC). Tumors were considered HER $2^{+}$only if they were scored $3+$ by IHC (strong, complete membranous staining in $>30 \%$ of cancer cells) and showed HER2 amplification (ratio >2) using fluorescence in situ hybridization (FISH). In the absence of positive FISH data, tumors scored 2+ using IHC were considered negative for HER2.

Tumor staging used the seventh edition of the American Joint Committee on Cancer (AJCC) cancer staging manual (22).

End-points. As mentioned above, this report was designed as a phase II utility trial evaluating the performance and diagnostic accuracy of a high-energy gamma probe capable of processing $511 \mathrm{keV}$ photons of ${ }^{18} \mathrm{~F}$-labeled pharmaceuticals. We undertook this investigation to assess the efficacy of a combined perioperative and intraoperative approach for tumor detection and evaluation of locoregional nodal disease in breast cancer. Technical performance was defined by the effect that use of the probe had on surgical exploration, according to both clinical parameters and breast cancer subtype, in informing treatment decisions for these categories. 


\section{Results}

Patient 1. The first case involved an 81-year-old Caucasian woman with locally advanced disease in the right breast (PT4) who underwent a radical mastectomy and ipsilateral ALND. Clinical examination revealed a $5 \mathrm{~cm}$ palpable mass in the upper-outer quadrant and no palpable right axillary mass. These findings were confirmed using mammography and ultrasound, and a subsequent 14-gauge core biopsy revealed invasive ductal carcinoma (IDC).

On the day of surgery, the preoperative clinical PET/CT demonstrated a solitary hypermetabolic lesion within the right breast (Figure 1). The $\mathrm{SUV}_{\max }$ of the lesion was 5.07 while the MTV was $20.24 \mathrm{~cm}^{3}$. No additional hypermetabolic lesions were found.

The patient was subsequently taken to the operating theatre. Intraoperative verification of the tumor location within the right breast and right axilla was performed by palpation and by handheld gamma probe detection. After careful analysis of the axillary tissue, any pathological uptake was detected as the surgical bed and was found to have no activity above background. Pathology revealed a $5.5 \mathrm{~cm}$ moderately differentiated invasive carcinoma with skin ulceration and seventh axillary lymph nodes without metastatic disease (pN0). Furthermore, the prognostic and predictive factors assessment showed a luminal B HER2profile (ER 95\%, PR 80\%, 40\% Ki-67, HER2 score 1), according to the St. Gallen Consensus 2013 (21).

In this case, the experimental procedure revealed a good diagnostic performance, with interesting clinical implications allowing for a more conservative surgical approach on the basis of ${ }^{18} \mathrm{~F}$-FDG PET/CT scanning, and the potential reduction of short- and long-term adverse events.

Patient 2. A 68-year-old Caucasian woman was originally diagnosed with well differentiated and multifocal IDC (PT1c) of her right breast before undergoing a rotational advancement flap quadrantectomy and ipsilateral ALND. On the day of surgery, the preoperative clinical PET/CT revealed two hypermetabolic foci in the lower-outer quadrant, with an $\mathrm{SUV}_{\text {max }}$ and MTV of 4.14 and $2.86 \mathrm{~cm}^{3}$, respectively, for the first lesion, and an 5.02 and $1.12 \mathrm{~cm}^{3}$, respectively, for the second. Additionally, a solitary hypermetabolic lymph node in the right axilla was found with an SUVmax of 2.38 and MTV of $782 \mathrm{~mm}^{3}$ (Figure 2). Intraoperative verification of the tumor location within the breast and the axillary area was performed by palpation and by handheld gamma probe detection, allowing improved location of the lymphatic metastasis present on preoperative imaging (Figure 3). After the resection, the surgical bed was found to have no activity above the background level. Pathological evaluation confirmed the tumor multifocality, identifying two lesions of $1.6 \mathrm{~cm}$ and $0.5 \mathrm{~cm}$, and negative surgical margins. Moreover, in the lymphatic structure removed following detection by probe, two axillary lymph nodes showed evidence of metastatic disease, and further nodal micrometastases was identified among the five nodes present in the remaining tissue from axillary dissection ( $\mathrm{pN} 1 \mathrm{a})$. The prognostic and predictive factor assessment showed a luminal A immunophenotypic profile (ER 95\%, PR 90\%, 5\% Ki-67, HER2 score 2), according to the St. Gallen Consensus 2013 (21). In this patient, the PET/CT scan had good diagnostic accuracy in identifying a multifocal breast lesion, in addition to a reasonable sensitivity for preoperative identification of pathological axillary involvement.

However, the spatial and topographic acquisition of the hypermetabolic lymphatic structure and its subsequent resection by handheld gamma probe detection were more complex and elaborate than the traditional method, demonstrating the need for further modifications for optimal technical performance. In conclusion, the procedure was useful in carrying out radio-guided selective axillary dissection, although it has low accuracy in the identification of nodal micrometastasis, for which there are little data about the short- and long-term prognostic value.

Patient 3. A 87-year-old Caucasian woman was originally diagnosed with locally advanced disease in the left breast (PT4) and underwent radical mastectomy and ipsilateral ALND. On the day of surgery, the preoperative clinical PET/CT demonstrated multiple hypermetabolic lesions of the inner quadrants with an SUVmax of 5.67 and an MTV of $23.81 \mathrm{~cm} 3$, in association with lymphangitic carcinomatosis (Figure 4). PET/CT scan also revealed centimetric lymph nodes in the left axilla that were not characterized by significant FDG uptake $\left(\mathrm{SUV}_{\max }\right.$ node $<\mathrm{SUV}_{\max }$ liver $)$. Intraoperative verification of tumor location within the breast and left axilla was performed by palpation and by handheld gamma probe detection, revealing widespread pathological uptake in the nodal area. Pathology revealed multifocal and multicentric breast disease, identifying three lesions of $8 \mathrm{~cm}$, $7 \mathrm{~cm}$, and $0.3 \mathrm{~cm}$, in association with angioinvasion and perineural invasion. Furthermore, 16 out of 17 axillary nodes had metastatic disease, and only one was positive for micrometastases, ( $\mathrm{pN} 3 \mathrm{a})$. The histological classification of the primary tumor demonstrated sections of poorly differentiated IDC, while the prognostic and predictive factors evaluation showed a luminal B HER2 ${ }^{-}$profile $\left(95 \%\right.$ ER, $\mathrm{PR}^{-}, \mathrm{Ki}-67$ 20\%, HER2 score 0), according to the Consensus of St. Gallen, 2013 (21). In this case the handheld gamma probe procedure revealed a good diagnostic accuracy in detecting a multifocal lesion, in addition to low sensitivity in the preoperative definition of pathological axillary involvement. However, despite the absence of specific nodal uptake by preoperative PET/CT imaging, the application of the handheld gamma probe provided real-time complementary data that aided in the definition of pathological tissues. 

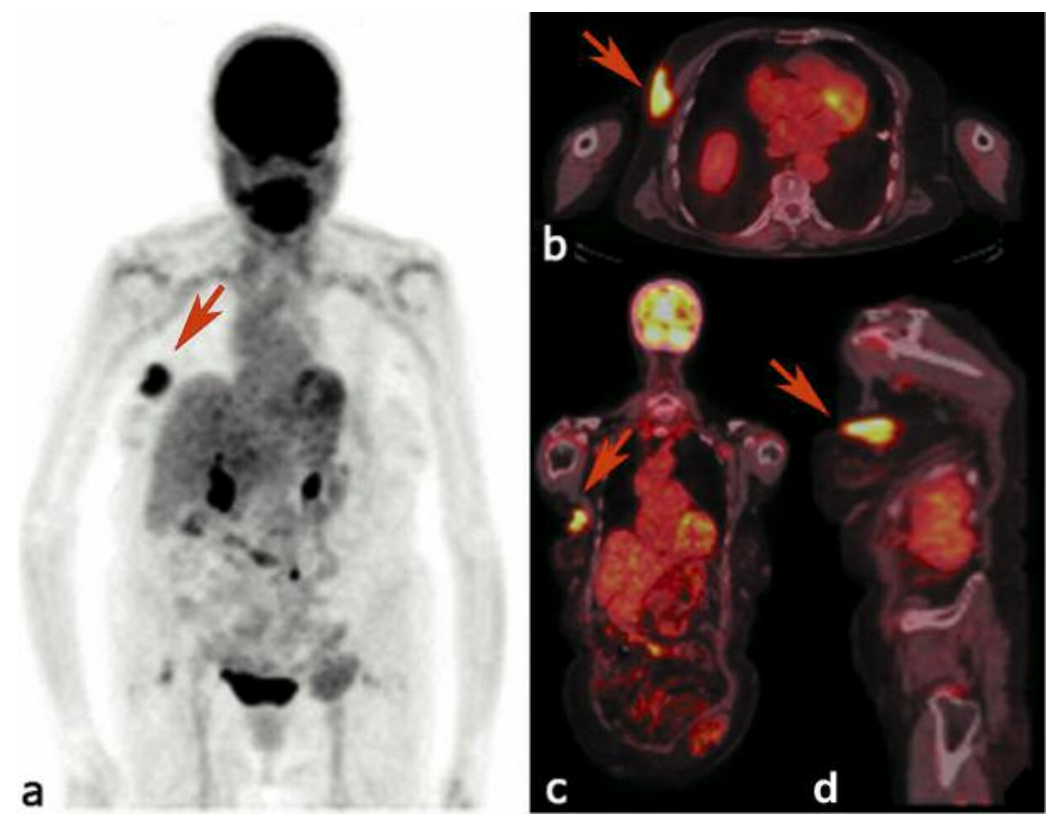

Figure 1. a: Maximum intensity projection of positron-emission tomography images showing the ${ }^{18} \mathrm{~F}$ - fluorodeoxyglucose distribution in patient 1 . Axial (b), coronal (c) and sagittal (d) positron-emission tomography/computed tomographic images demonstrating a hypermetabolic lesion in the upper-outer quadrant of right breast (red arrows).

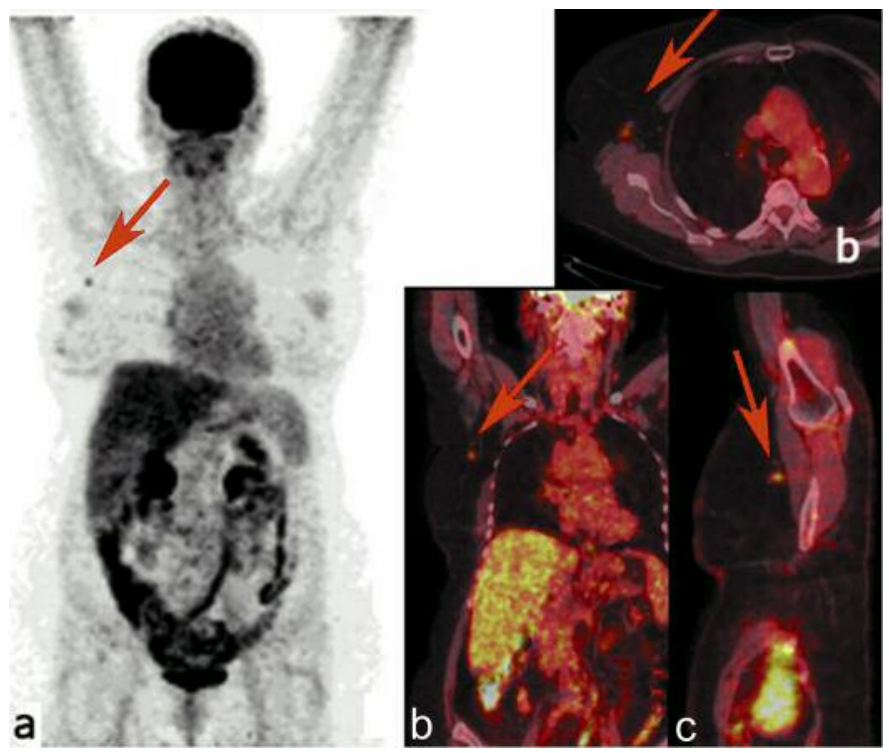

Figure 2. Maximum intensity projection of positron-emission tomography images showing the ${ }^{18} \mathrm{~F}$ - fluorodeoxyglucose distribution in patient 2 (a). Axial $(b)$, coronal $(c)$ and sagittal $(d)$ positron-emission tomography/computed tomographic images demonstrating a hypermetabolic lymph node in the right axilla (red arrows).

\section{Discussion}

It is well-established that ${ }^{18} \mathrm{~F}-\mathrm{FDG}$ PET imaging is a powerful tool for assisting in the diagnosis, staging, and monitoring of therapy response for a variety of neoplastic processes, including breast cancer $(23,24)$. One of the main limitations of the current use of PET imaging is that our standard practice of preoperative image acquisition at the time of the original evaluation of the patient cannot be translated into real-time information for availability in the 

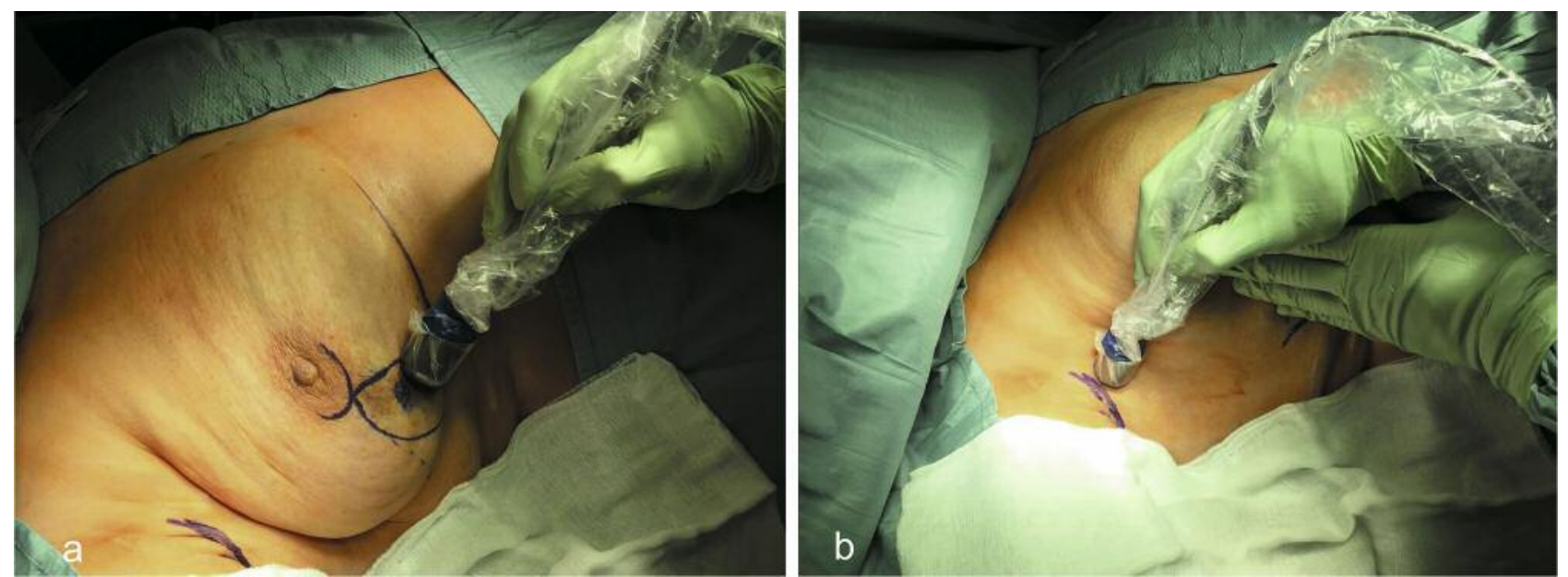

Figure $3 .{ }^{18}$ F-Fluorodeoxyglucose $\left({ }^{18} \mathrm{~F}\right.$-FDG)-handheld gamma probe detection for tumor localization in the right breast (a); positron-emission tomography probe-guided excision of an ${ }^{18}$ FDG-positive lymph node in the axilla (b). Final pathology confirmed nodal metastases.

operating theatre. It can often be difficult for the oncological surgeon to intraoperatively localize presumed abnormalities that were evident on preoperative image acquisition. Such limitations can result in the inability to detect occult disease and may lead to incomplete tumor resection during definitive cancer surgery. This may ultimately be responsible for disease recurrence and impact negatively upon long-term patient outcomes. Thus, by allowing the oncological surgeon access to imaging and metabolic information intraoperatively using perioperative ${ }^{18} \mathrm{~F}-\mathrm{FDG}$ PET/CT imaging and intraoperative ${ }^{18} \mathrm{~F}$-FDG gamma probe detection has great potential to significantly improve the overall success of complete surgical resection, and may ultimately positively influence long-term patient outcomes.

There is an ongoing debate regarding the potential role of ${ }^{18}$ F-FDG PET for initial staging of breast cancer $(25,26)$. Despite a low sensitivity, ${ }^{18} \mathrm{~F}$-FDG PET/CT is generally reported to have a good specificity for identifying axillary nodal disease. Some authors advocate the use of ${ }^{18}$ F-FDG $\mathrm{PET} / \mathrm{CT}$ in order to reduce the use of SNB, evaluating patients with a high axillary lymph node burden who could then move directly to ALND (27). Others suggest that ${ }^{18}$ F-FDG PET/CT should be used to extend the use of SNB over more invasive methods, since in patients with a high risk of axillary lymph node metastases that are not considered candidates for SNB, an unremarkable ${ }^{18} \mathrm{~F}-\mathrm{FDG}$ PET/CT scan can still identify those who can safely undergo SNB instead of immediate ALND. Such an approach may extend the indication for SNB to patients with an up to $60 \%$ risk of lymph node metastases (corresponding to a tumor diameter of $4-5 \mathrm{~cm}$ ) and thus avoid the comorbidity of unnecessary ALNDs in a substantial proportion of patients (28). However, management of the axilla in patients with operable breast cancer is still one of most controversial areas in clinical oncology. A recent systematic review found that the sensitivity of PET/CT systems in the detection of axillary nodal metastases ranged from $44 \%$ to $67 \%$ (29). Patients with false-negative PET results had significantly smaller and fewer tumor-positive lymph nodes than true-positive cases. The results indicated that there are limitations to FDG PET in the detection of micrometastases and small, tumor-infiltrated axillary lymph nodes (30). This low sensitivity is due, in part, to the limited spatial resolution (approximately 5-6 mm) of PET systems, leading to partial volume effects that cause significant underestimation of the radioactivity concentration in lesions smaller than 2-3 times the spatial resolution of the systems.

Concerning this issue, further work is required to address issues of sensitivity and specificity. Both the size of the lesion and the intensity of tracer uptake are important determinants for lesion detectability with PET. Phantom studies show that lesions $<5 \mathrm{~mm}$ are not reliably detected on PET (30). Thus it is not surprising that micrometastases $(<2 \mathrm{~mm})$ are not detected, and indeed a recent technological assessment showed that the smallest axillary node detected using PET was $3 \mathrm{~mm}$ (31). The sensitivity for detection of axillary nodal metastasis can be improved by reducing the SUV threshold, but this can negatively impact specificity. Therefore, low-grade ${ }^{18}$ F-FDG nodal uptake may simply represent inflammatory (reactive) changes and thus give rise to false-positive findings.

Despite this, evaluation of new technologies that are being implemented in PET imaging is needed. Some have such advanced reconstruction algorithms that they are likely not only to improve diagnostic performance, and thus the 

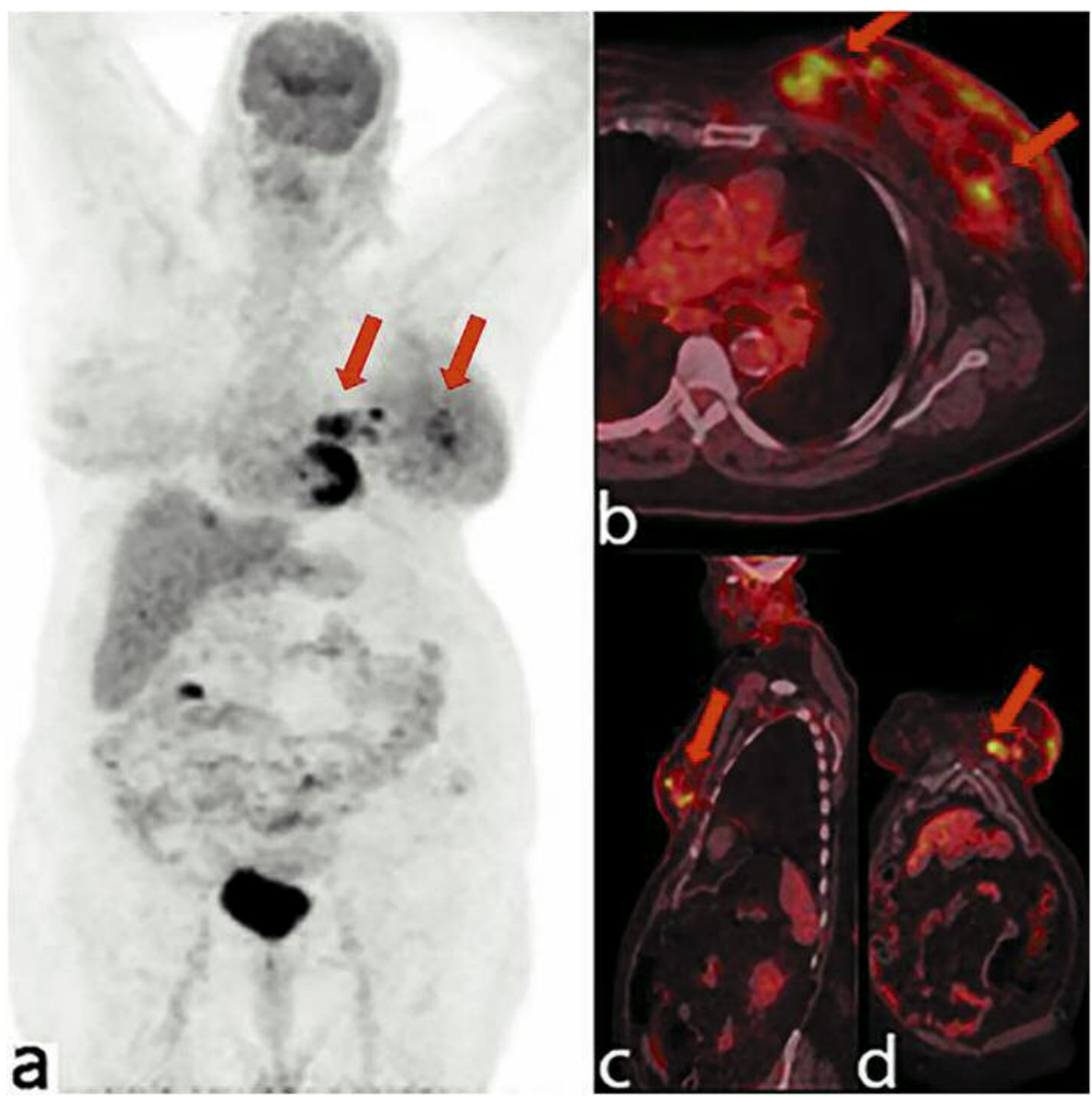

Figure 4. Maximum intensity projection of positron-emission tomography. Images showing the ${ }^{18} \mathrm{~F}$ - fluorodeoxyglucose distribution in patient 3 (a). Axial $(b)$, coronal $(c)$, and sagittal $(d)$ positron-emission tomography/computed tomographic images demonstrating multiple hypermetabolic lesions in the left breast, in association with lymphangitic carcinomatosis (red arrows).

detectability of smaller lymph node metastases (32-33), but also to change quantitative and imaging features, requiring new diagnostic thresholds to be defined (34).

Moreover, the added value of PET in breast cancer staging is that it is a non-invasive technique that allows the study of all lymphatic basins of the breast (axilla, supraclavicular and mammary chain), and in a single examination permits both the characterization of breast lesions and the complete viewing of the entire body. This means that we have at our disposal a single radiopharmaceutical and a single diagnostic modality for the complete evaluation of all possible sites of breast cancer metastasis in order to characterize their prognostic effects over time.

In this context, the clinical significance of microscopic lymph node involvement not detected by ${ }^{18} \mathrm{~F}-\mathrm{FDG}$ PET/CT was also analyzed during follow-up for relapse and distant metastases. A recent study suggested that axillary node metastases which were not visualized by ${ }^{18}$ F-FDG PET /CT seemed to have little clinical significance for relapse or distant metastases (35). Indeed, few retrospective analyses on selected patients with SLN micrometastases without further ALND have suggested that this subset of patients will suffer from a higher incidence of regional recurrence or distant disease. This supports the theory that formal ALND may be omitted in these patients, thus reducing the potential associated short- and long-term complications (3638). However, the design of a probe that is capable of processing high-energy gamma photons from positron emitters such as ${ }^{18} \mathrm{~F}$ and using it intraoperatively with significant background radiation is highly challenging. The ability of a gamma probe to identify a lesion depends on several factors, including the FDG avidity of the tumor, the timing of surgical exploration related to the injection of FDG, the anatomical location of the lesion, and its relative proximity to the main sites of physiological uptake. All these features are major determinants of the clinical 
performance of a gamma probe, and differences in these properties are likely to lead to variable results in surgery. This study demonstrates the technical ability and feasibility of a handheld PET probe for lesion detection, and in one case, it was useful in localizing additional disease not detected on the preoperative imaging study.

Nevertheless, the present study has several inherent limitations. It was an experimental proposal for a methodological approach, and our sample size was small. Therefore, no statistical analysis was performed, resulting in restrictions in interpreting the findings. Clinical outcomes are an important end-point for this methological approach. In this respect, no relapse or metastases were found in an average follow-up period of $10.42 \pm 10.56$ months in patients with axillary metastases and micrometastasis on an ${ }^{18} \mathrm{~F}-\mathrm{FDG}$ PET/CT scan. A larger patient population would have been beneficial but this would have significantly increased the recruitment and follow-up periods. A longer follow-up period would also have been ideal but this had to be balanced against the need to obtain data that are lacking in the current literature. Furthermore, our team's limited experience and familiarity with PET probe-guided surgery may have influenced our outcomes. However, our findings highlight the importance of a recent call for a randomized, controlled trial to further investigate the role of PET/CT in breast cancer staging (39).

Another limitation of this study is that the three patients were 68,81 , and 87 years old and not representative of the population of patients with breast cancer. The peak age for breast tumors is between 40 and 50 years in Asian countries, whereas the peak age in Western countries is between 60 and 70 years (40). However, recent data suggest that although breast cancer outcomes in younger women have shown substantial improvement because of advances in treatment and screening, the benefits in older women have been less pronounced. In addition, planning therapy for older patients is not always straightforward because they may present comorbidities and frailty that limit therapeutic choices. In this context, the exclusion of node involvement using noninvasive methods could reduce the rate of axillary surgery, thereby preventing complications in a substantial proportion of patients. The results of this study suggest FDG PET/CT could be used as a initial test prior to invasive staging procedures, but further trials with a large number of patients need to be performed to verify our findings.

Currently, with known limitations, FDG imaging represents the standard for functional and biological imaging in oncology (41). Many more new PET radiopharmaceuticals are being developed for distinct tumor types and metabolic properties (42). In future, the clinical indications for PET probe use will be individually determined on the basis of tumor and patient characteristics, as well as the distribution patterns of the selected PET for the desired imaging targets (radiopharmaceuticals). Further explorations of the feasibility of these techniques, as well as correlation with pathology and patient outcomes, will be helpful in assessing the benefit of these procedures.

\section{Conclusion}

Although ${ }^{18}$ F-FDG PET/CT is valuable for detecting distant metastases, identifying recurrence, and evaluating response to chemotherapy, its role in evaluating locoregional nodal status for initial staging of breast cancer has not yet been well-defined in clinical practice. Thus, the availability of an innovative combined imaging/detection technology in the perioperative and intraoperative arena has the potential for improving oncological surgeons' success rates in achieving a complete tumor resection. The current report demonstrates great promise for ${ }^{18} \mathrm{~F}-\mathrm{FDG} \mathrm{PET} / \mathrm{CT}$ in providing real-time access to metabolic and morphological tumor information that may lead to an optimal disease-tailored approach.

However, in our study, the role of ${ }^{18} \mathrm{~F}$-FDG PET/CT in axillary node staging was limited, suggesting that for the assessment of regional nodal metastases, surgical biopsy and histological analysis remain indicated. ${ }^{18}$ F-FDG PET was poor at providing effective evaluation of axillary lymph nodes, but future hardware and software improvements might improve the spatial resolution for detecting locoregional disease, allowing for a selective approach to diagnose metastases in axillary nodes and SLN. As suggested by previous reports, these false-negative results may be due to the small size of the axillary node, stromal-rich cancer, positive lymphovascular invasion, multifocal tumors, and high tumor staging $(43,44)$. Consequently, the true incidence of ${ }^{18}$ F-FDG PET-detectable disease in various stages of primary breast cancer needs to be determined and systematically measured. Subsequently, the way in which this detection method alters patient management, survival, and quality of life should be assessed. Furthermore, the potential for a more widespread application of this innovative technology is likely to lie in applications such as verification of primary tumor site resection, margin determination, regional lymph node assessment, and recognition of sites of occult disease.

The limitations of PET scanning, as well as the lack of specificity of ${ }^{18}$ F-FDG as a tumor tracer, are acknowledged. However, with further development of new diagnostic thresholds, all of these potential applications may ultimately positively influence long-term patient outcomes.

\section{Competing Interests}

No benefits in any form have been received or will be received from any commercial party related directly or indirectly to the subject of this article. 


\section{Funding}

This research project was conducted in collaboration with the Italian Ministry of Health.

\section{References}

1 Lale Atahan I, Yildiz F, Ozyigit G, Sari S, Gurkaynak M, Selek $\mathrm{U}$ and Hayran M: Percent positive axillary lymph node metastasis predicts survival in patients with non-metastatic breast cancer. Acta Oncol 47(2): 232-238, 2008.

2 Truong PT, Berthelet E, Lee J, Kader HA and Olivotto IA: The prognostic significance of the percentage of positive/dissected axillary lymph nodes in breast cancer recurrence and survival in patients with one to three positive axillary lymph nodes. Cance 103(10): 2006-2014, 2005.

3 Voordeckers M, Vinh-Hung V, Van de Steene J, Lamote J and Storme G: The lymph node ratio as prognostic factor in nodepositive breast cancer. Radiother Oncol 70(3): 225-230, 2004

4 Megale Costa LJ, Soares HP, Gaspar HA, Trujillo LG, Santi PX, Pereira RS, de Santana TL, Pinto FN and del Giglio A :Ratio between positive lymph nodes and total dissected axillaries lymph nodes as an independent prognostic factor for disease free survival in patients with breast cancer. Am J Clin Oncol 27(3): 304-306, 2004.

5 van der Wal BC, Butzelaar RM, van der Meij S and Boermeester MA: Axillary lymph node ratio and total number of removed lymph nodes: Predictors of survival in stage I and II breast cancer. Eur J Surg Oncol 28(5): 481-489, 2002.

6 Lyman GH, Temin S, Edge SB, Newman LA, Turner RR, Weaver DL, Benson AB 3rd, Bosserman LD, Burstein HJ, Cody H 3rd, Hayman J, Perkins CL, Podoloff DA, Giuliano AE; American Society of Clinical Oncology Clinical Practice: Sentinel lymph node biopsy for patients with early-stage breast cancer: American Society of Clinical Oncology clinical practice guideline update. J Clin Oncol 32: 1365-1383, 2014.

7 Oliveira M, Cortés J, Bellet M, Balmaña J, De Mattos-Arruda L, Gómez P, Muñoz E, Ortega V, Pérez J, Saura C, Vidal M, Rubio IT and Di Cosimo S: Management of the axilla in early breast cancer patients in the genomic era. Ann Oncol 24(5): 1163-1170, 2013

8 Wang M, Zhou W, Zhao Y, Xia T, Zha X, Ding Q, Liu X, Zhao Y, Ling L, Chen L and Wang S: A novel finding of sentinel lymphatic channels in early stage breast cancer patients: which may influence detection rate and false-negative rate of sentinel LymphNode Biopsy. PLoS ONE 7(12): e51226, 2012.

9 Suami H, Pan WR, Mann GB and Taylor GI: The lymphatic anatomy of the breast and its implications for sentinel lymph node biopsy: a human cadaver study. Ann Surg Oncol 15(3): 863-871, 2008.

10 Groves AM, Wishart GC, Shastry M, Moyle P, Iddles S, Britton P, Gaskarth M, Warren RM, Ell PJ and Miles KA: Metabolicflow relationships in primary breast cancer: feasibility of combined PET/dynamic contrast-enhanced CT. Eur J Nucl Med Mol Imaging 36(3): 416-421, 2009

11 Buck A, Schirrmeister H, Kühn T, Shen C, Kalker T, Kotzerke J, Dankerl A, Glatting G, Reske S and Mattfeldt T: FDG uptake in breast cancer: correlation with biological and clinical prognostic parameters. Eur J Nucl Med Mol Imaging 29(10): 1317-1323, 2002.
12 Buscombe JR, Holloway B, Roche N and Bombardieri E: Position of nuclear medicine modalities in the diagnostic work-up of breast cancer. Q J Nucl Med Mol Imaging 48(2): 109-118, 2004.

13 Gulec SA, Hoenie E, Hostetter R and Schwartzentruber D: PET probe-guided surgery: applications and clinical protocol. World J Surg Oncol 5: 65, 2007.

14 Orsaria P, Varvaras D, Vanni G, Pagnani G, Scaggiante J, Frusone F, Granai AV, Petrella G and Buonomo OC: Nodal status assessment in breast cancer: strategies of clinical grounds and quality of life implications. Int J Breast Cancer 2014: 469803, 2014.

15 Buonomo OC, Orsaria P, Pagnani G, Scaggiante J, Rossi P, Venditti D, Granai AV, Vanni G, Varvaras D,Esser A, Petrella G and Palombi L: Biological relevance of clinical and pathological features in breast cancer molecular subtypes: additional insights for personalized decision-making in nodal status assessment. IJSR 4: 128-133, 2015.

16 Chiaravalloti A, Danieli R, Caracciolo CR, Travascio L, Cantonetti M, Gallamini A, Guazzaroni M, Orlacchio A, Simonetti G, Schillaci O:Initial staging of Hodgkin's disease: role of contrast-enhanced ${ }^{18} \mathrm{~F}$ FDG PET/CT. Medicine 93: e50, 2014.

17 Desai D, Arnold M, Saha S, Hinkle G, Soble D, Fry J, DePalatis LR, Mantil J, Satter M and Martin EW: Intraoperative Gamma Detection of FDG Distribution in Colorectal Cancer. Clin Positron Imaging 3(5): 189-196, 2000.

18 Nwogu C, Fischer G, Tan D, Glinianski M, Lamonica D, Demmy $\mathrm{T}$ : Radioguided detection of lymph node metastasis in nonsmall cell lung cancer. Ann Thorac Surg 82: 1815-1820, 2006.

19 Boerner AR, Weckesser M, Herzog H, Schmitz T, Audretsch W, Nitz U, Bender HG and Mueller-Gaertner HW: Optimal scan time for fluorine-18 fluorodeoxyglucose positron-emission tomography in breast cancer. Eur J Nucl Med 26: 226-230, 1999.

20 Kuker RA, Mesoloras G and Gulec SA: Optimization of FDG$\mathrm{PET} / \mathrm{CT}$ imaging protocol for evaluation of patients with primary and metastatic liver disease. Int Semin Surg Oncol 4: 17, 2007.

21 Goldhirsch A, Winer EP, Coates AS, Gelber RD, Piccart-Gebhart M, Thürlimann B, Senn HJ; Panel members: Personalizing the treatment of women with early breast cancer: highlights of the St Gallen International Expert Consensus on the Primary Therapy of Early Breast Cancer 2013. Ann Oncol 24(9): 22062223, 2013.

22 Edge SB, Byrd DR, Compton CC, Fritz AG, Greene FL and Trotti A: AJCC Cancer Staging Manual (7th ed). New York, NY: Springer; 2010.

23 Iagaru A, Masamed R, Keesara S and Conti PS: Breast MRI and ${ }^{18} \mathrm{~F}$ FDG PET/CT in the management of breast cancer. Ann Nucl Med 21: 33-38, 2007.

24 Tatsumi M, Cohade C, Mourtzikos KA, Fishman EK, Wahl RL: Initial experience with FDG-PET/CT in the evaluation of breast cancer. Eur J Nucl Med Mol Imaging 33: 254-262, 2006.

25 Aukema TS, Straver ME, Valdes Olmos RA and Vogel WV: A different role for FDG PET/CTin axillary lymph node staging in breast cancer. Eur J Nucl Med Mol Imaging 36: 1896-1897, 2009.

26 Chua S and Groves AM: Pretreatment PET in breast cancer: is there a role? Eur J Nucl Med Mol Imaging 39: 1827-1829, 2012.

27 Liu Y: Role of FDG PET-CT in evaluation of locoregional nodal disease for initial staging of breast cancer. World J Clin Oncol 5: 982-989, 2014. 
28 Heusner TA, Kuemmel S, Hahn S, Koeninger A, Otterbach F, Hamami ME, Kimmig KR, Forsting M, Bockisch A, Antoch G and Stahl A: Diagnostic value of full-dose FDG PET/CT for axillary lymph node staging in breast cancer patients. Eur J Nucl Med Mol Imaging 36: 1543-1550, 2009.

29 Cooper KL, Harnan S, Meng Y, Ward SE, Fitzgerald P, Papaioannou D, Wyld L, Ingram C, Wilkinson ID and Lorenz E: Positron emission tomography (PET) for assessment of axillary lymph node status in early breast cancer: a systematic review and meta-analysis. Eur J Surg Oncol 37: 187-198, 2011.

30 Wahl RL, Siegel BA, Coleman RE, Gatsonis CG; PET Study Group: Prospective multicenter study of axillary nodal staging by positron emission tomography in breast cancer: a report of the staging breast cancer with PET Study Group. J Clin Oncol 22: 277-285, 2004.

31 Cooper KL, Meng Y, Harnan S, Ward SE, Fitzgerald P, Papaioannou D, Wyld L, Ingram C, Wilkinson ID and Lorenz E: Positron-emission tomography (PET) and magnetic resonance imaging (MRI) for the assessment of axillary lymph nodemetastases in early breast cancer: Systematic review and economic evaluation. Health Technol Assess 15: 1-134, 2011.

32 Lewellen TK: Recent developments in PET detector technology. Phys Med Biol 53: R287-317, 2008.

33 Teräs M, Tolvanen T, Johansson JJ, Williams JJ and Knuuti J: Performance of the new generation of whole-body PET/CT scanners: Discovery STE and Discovery VCT. Eur J Nucl Med Mol Imaging 34: 1683-1692, 2007.

34 Bellevre D, Blanc Fournier C, Switsers O, Dugué AE, Levy C, Allouache D, Desmonts C, Crouet H, Guilloit JM, Grellard JM and Aide N: Staging the axilla in breast cancer patients with ${ }^{18} \mathrm{~F}-$ FDG PET: How small are the metastases that we can detect with new generation clinical PET systems? Eur J Nucl Med Mol Imaging 41: 1103-1112, 2014.

35 Zhang X, Wu F and Han P: The role of (18)F-FDG PET/CT in the diagnosis of breast cancer and lymph nodes metastases andmicrometastases may be limited. Hell J Nucl Med 17(3): 177-183, 2014.

36 Fant JS, Grant MD, Knox SM, Livingston SA, Ridl K, Jones RC and Kuhn JA: Preliminary outcome analysis in patients with breast cancer and a positive sentinel lymph node who declined axillary dissection. Ann Surg Oncol 10: 126-130, 2003.
37 Guenther JM, Hansen NM, DiFronzo LA, Giuliano AE, Collins JC, Grube BL and O'Connell TX: Axillary dissection is not required for all patients with breast cancer and positive sentinel nodes. Arch Surg 138: 52-56, 2003.

38 Liang WC, Sickle-Santanello BJ and Nims TA: Is a completion axillary dissection indicated for micrometastases in the sentinel lymph node? Am J Surg 182: 365-368, 2001.

39 Groves AM, Shastry M, Ben-Haim S, Kayani I, Malhotra A, Davidson T, Kelleher T, Whittaker D, Meagher M, Holloway B, Warren RM, Ell PJ and Keshtgar MR: Defining the Role of PET-CT in Staging Early Breast Cancer. Oncologist 17(5): 613619, 2012.

40 Curado MP: Breast cancer in the world: Incidence and mortality. Salud Publica Mex 53: 372-384, 2011.

41 Schillaci O: Pet Probes and oncological surgery: a productive new marriage for nuclear medicine? Eur J Nucl Med Mol Imaging 34: 1530-1533, 2007.

42 Gulec SA, Daghighian F and Essner R: PET-Probe: Evaluation of technical performance and clinical utility of a handheld highenergy gamma probe in oncologic surgery. Ann Surg Oncol 23: 9020-9027, 2006.

43 Ueda S, Tsuda H, Asakawa H, Omata J, Fukatsu K, Kondo N, Kondo T, Hama Y, Tamura K, Ishida J, Abe Y and Mochizuki H: Utility of ${ }^{18} \mathrm{~F}$-fluoro-deoxyglucose emission tomography/ computed tomography fusion imaging $\left({ }^{18} \mathrm{~F}\right.$-FDGPET/CT) in combination with ultrasonography for axillary staging in primary breast cancer. BMC Cancer 8: 165, 2008

44 Zornoza G, Garcia-Velloso MJ, Sola J Regueira FM, Pina L and Beorlegui C: ${ }^{18} \mathrm{~F}-\mathrm{FDG}$ PET complemented with sentinel lymph node biopsy in the detection of axillary involvement in breast cancer. EurJ Surg Oncol 30: 15-19, 2004. 